\title{
The Interaction of Dose and Rate of Delivery Using Visible and Infrared Light to Inhibit Pseudomonas aeruginosa In vitro
}

\section{J. Stephen Guffey ${ }^{1^{*}}$, William Payne ${ }^{2}$ and Annette Bednar ${ }^{2}$}

${ }^{1}$ Department of Physical Therapy, Arkansas State University, State University, Arkansas, USA

${ }^{2}$ Department of Clinical Laboratory Science, Arkansas State University, State University, Arkansas, USA

*Corresponding author: J. Stephen Guffey, Department of Physical Therapy, Arkansas State University, State University, Arkansas, USA, Tel: 870-972-3591; E-mail: jguffey@astate.edu

Rec date: Feb 14, 2014; Acc date: Apr 17, 2014; Pub date: Apr 23, 2014

Copyright: ( 2014 Guffey JS, et al. This is an open-access article distributed under the terms of the Creative Commons Attribution License, which permits unrestricted use, distribution, and reproduction in any medium, provided the original author and source are credited

\begin{abstract}
Background: Visible and near-IR energies have been shown to inhibit the growth of various microorganisms. There may be clinical application for this inhibitory effect in the treatment of infected wounds particularly, and other infectious states generally.
\end{abstract}

Aim: The purpose of this study was to determine whether there was an interactive effect between dose and rate of energy delivery when using combined 464 and $850 \mathrm{~nm}$ light to inhibit Pseudomonas aeruginosa.

Methods: Solutions containing $P$. aeruginosa was irradiated with blue and infrared light (464 and $850 \mathrm{~nm})$. The light energy was applied at two different doses and at two different rates of delivery. The doses employed included 9 and $30 \mathrm{Jcm}^{-2}$. The rates of delivery used were 20 and $125 \mathrm{mWcm}^{-2}$

Design: An in vitro $2 \times 2$ factorial design was used to compare doses of 9 and $30 \mathrm{Jcm}^{-2}$ and rates delivery of $20 \mathrm{~m}$ $\mathrm{Wcm}^{-2}$ and $125 \mathrm{mWcm}^{-2}$ to determine kill rates compared to controls. Each dose was applied at each delivery rate.

Results: Student t-tests demonstrated $(p=0.00)$ a significant kill rate at three of the four combinations. A two-way analysis of variance identified a significant interactive effect between dose and rate $(F=94.717, p=0.00)$. Tukey's HSD identified $30 \mathrm{Jcm}^{-2}$ delivered at a $20 \mathrm{mWcm}^{-2}$ as significantly different from the other combinations.

Conclusions: These data suggest that 1) visible and infrared light may be effective inhibitors of $P$. aeruginosa, and 2) both dose and rate of delivery are important factors to consider when using visible and infrared light energy to inhibit the growth of this organism.

Keywords: Bactericidal; Biostimulation; Biomodulation

\section{Introduction}

Antimicrobial resistance to drug therapy has emerged as one of the major challenges facing clinicians tasked with treating skin breakdown $[1,2]$. Pharmaceutical agents used to treat infection have made a significant contribution to human health [3], but demonstrated drug resistance threatens the long term facility of antibiotics. As Bush et al. [4] have pointed out, finding alternative antimicrobial agents/ techniques must be a high-priority focus of current research. To this end, prior to this current experiment, we have been engaged in examining the bactericidal [5-9] and fungicidal [10] effects of visible and infrared light.

Blue light (405-470 $\mathrm{nm}$ ) has been shown to be an effective in vitro inhibitor of Staphylococcus aureus and Pseudomonas aeruginosa [5]. Combining near infrared $(850-880 \mathrm{~nm})$ wavelengths with blue light can enhance in vitro bacterial inhibition [6]. Additionally, the use of light to treat infection in skin lesions in live animal models has been supported. Dai, Gupta and Huang et al. [2] recently demonstrated 405 $n m$ light was effective in inhibiting $P$. aeruginosa in vivo related to mouse burn wounds.
While higher doses (compared to those needed with $S$. aureus and $P$. aeruginosa) of light energy are required for effective inhibition of Mycobacterium smegmatis, blue light is an effective bactericidal agent against this organism $[7,11,12]$. Visible (primarily blue) light has also been shown to be an effective inhibitor of other microorganisms [13-15].

Since the basis for visible light as an inhibitor of bacterial growth was being established in the literature, we turned our attention to whether bacteria might develop resistance to repeated applications of light energy. We did demonstrate this potential with regard to S. aureus [8]. Subsequently, we developed a technique to delay the expression of this resistance [9]. In the process of seeking ways to delay the expression of resistance, we found that rate of energy delivery (not just wavelength or dose) appeared to have an effect on outcome. This point was made some time ago by Wainwright, [16] but escaped our attention until we were faced with the issue of resistance formation in some of our earlier work [8]. While we have not established whether $P$. aeruginosa, like $S$. aureus, [8] is able to develop resistance to light energies previously demonstrated to be inhibitory, we suspect that possibility exists. This is why we chose to manipulate, in this current study, wavelength, dose, and rate of delivery. This current study does not address resistance expression, but does include 
energy deliver techniques we have learned in experiments related to resistance expression.

This study was undertaken to more completely examine the effect rate of energy delivery might have on inhibition of $P$. aeruginosa. We were also, of course, interested in the effect of dose, but this variable has been studied already as mentioned above. To achieve this goal, we employed an experimental design comparing Colony Forming Units (CFU) in control versus treated samples to determine a kill rate expressed in percentage ([Control CFU - Treated CFU]/Control CFU $\times 100$ ). More specifically, the design was a two-way factorial method. The first of the two independent variables was dose. Dose contained two levels $\left(9 \mathrm{Jcm}^{-2}\right.$ and $\left.30 \mathrm{Jcm}^{-2}\right)$. The second independent variable was rate of delivery. It also included two levels $\left(125 \mathrm{mWcm}^{-2}\right.$ and 20 $\mathrm{mWcm}^{-2}$ ). The dependent variable was the kill rate obtained. The questions we intended to answer were threefold. When delivering a combination of 464 and $850 \mathrm{~nm}$ light energy at 9 and $30 \mathrm{Jcm}^{-2}$ and at rates $125 \mathrm{mWcm}^{-2}$ and $20 \mathrm{mWcm}^{-2}$ ) is there a difference in bacterial kill rate based on dose (Main Effect), 2) is there a difference in bacterial kill rate based on rate of delivery (Main Effect), and/ or 3) is there an interactive effect between dose and rate of delivery in terms of kill rate (Interaction Effect)? Figure 1 is a graphical representation of the research design.

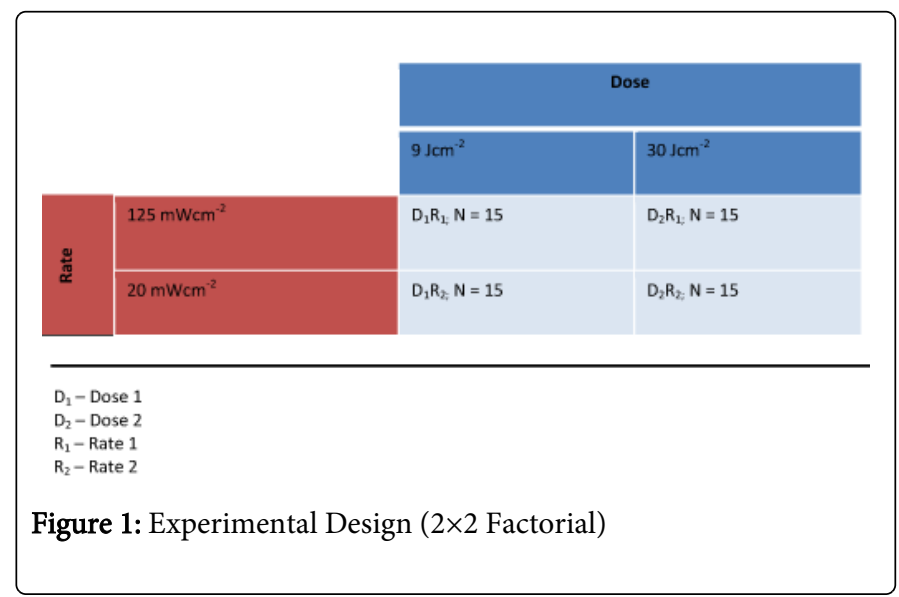

\section{Methods and Materials}

\section{Organism preparation}

The organism used for this study was Pseudomonas aeruginosa (ATCC 27853), a commonly encountered organism that thrives in wet environments such as sinks, faucets, and water baths. In the hospital environment, this organism has been recovered from respiratory equipment, medications, soaps, and may contaminate sterile solutions such as intravenous solutions. P. aeruginosa, an obligate aerobe, is not fastidious and grows well in ambient air on any type of nutritive medium [17]. Tryptic soy agar (DifcoTM, Detroit, USA), a general purpose medium, was chosen as the growth medium for subculture following exposure to light. The organism was then incubated at $37^{\circ} \mathrm{C}$ for a period of 20 hours. Use of a 20 -h-old culture is standard microbiological practice and serves to minimize the lag time for new growth.

Using a sterile cotton-tipped swab, material was removed from a Tryptic Soy Agar (TSA) plate and added to sterile deionized water to form a suspension equivalent to a $0.5 \mathrm{McF}$ arland standard. The suspension was then diluted $1 / 100$ using a 100 microliter automatic pipette (for purposes of accuracy and reproducibility). The 1/100 dilution was further diluted (1/100) to make a final dilution of $1 / 10,000$. All dilutions were made immediately before the administration of the light treatment (wavelengths of 464 and 850 $\mathrm{nm}$ ). Immediately following the light application, using a 10 microliter automatic pipette, an aliquot of the 1/10,000 dilution of $P$. aeruginosa was inoculated onto TSA in $100 \times 15 \mathrm{~mm}$ sterile, polystyrene petri dishes. The diluted bacterial suspension was applied to the surface of the TSA plates in a star-streak pattern to enable colony counts to be performed after approximately 24 hours of incubation.

Enumeration and quantitation of $P$. aeruginosa colonies is facilitated because this organism produces distinctive colonies that are flat, wrinkled, spreading, and have a "ground glass" appearance. These Colony Forming Units (CFUs) are readily distinguished from organisms that might appear as contaminants on non-selective media such as TSA plates.

\section{Light energy application}

The solution containing $P$. aeruginosa was subjected to blue and infrared light (464 and $850 \mathrm{~nm}$ ). The light energy was applied at two different doses and at two different rates of delivery. The doses employed included 9 and $30 \mathrm{Jcm}^{-2}$. The rates of delivery used were 20 and $125 \mathrm{mWcm}^{-2}$. A $2 \times 2$ factorial design was used in this study so that each dose was delivered at both the lower and the higher rate.

After each exposure and incubation at $37^{\circ} \mathrm{C}$ in ambient air, a colony count was performed to determine the kill rate. If the method of light exposure was effective in terms of slowing resistance formation, a consistent kill rate should have resulted across the seven stages.

Light exposures were achieved using The Dynatron ${ }^{*}$ 705Plus Solaris $^{\mathrm{Tm}}$ manufactured by Dynatronics Corp. (Salt Lake City, UT). This device is designed to accommodate both a light probe (higher rate of delivery, $125 \mathrm{mWcm}^{-2}$ ) and a pair of light pads (lower rate of delivery, $20 \mathrm{mWcm}^{-2}$ ). The probe consisted of a $5 \mathrm{~cm}^{2}$ illuminating surface area comprised of 34 Supra Luminous Diodes (SLDs) with a maximum power output of $1000 \mathrm{~mW}$. The pads consisted of a $353 \mathrm{~cm}^{2}$ total illuminating surface area comprised of 176 SLDs with a maximum power output of $5160 \mathrm{~mW}$. Dose was calculated in $\mathrm{Jcm}^{-2}$. Since output for the probe and the pads was held constant, adjustment in time of irradiation provided the dose $\left(9 \mathrm{Jcm}^{-2}\right.$ or $\left.30 \mathrm{Jcm}^{-2}\right)$. We chose the doses used in this experiment based on our previous work with various microbes [5-10]. These have consistently proven to be effective doses that can be easily employed clinically. Time to achieve the doses of 9 and $30 \mathrm{Jcm}^{-2}$ for each method of delivery is listed below (Table 1).

\begin{tabular}{|l|l|l|}
\hline & Probe & Pads \\
\hline $9 \mathrm{Jcm}^{-2}$ & 1 minute, 6 seconds & 8 minutes, 51 seconds. \\
\hline
\end{tabular}


Citation: Guffey JS, Payne W, Bednar A (2014) The Interaction of Dose and Rate of Delivery Using Visible and Infrared Light to Inhibit Pseudomonas aeruginosa In vitro. J Laser Opt Photonics 1: 104. doi:10.4172/2469-410X.1000104

Page 3 of 4

\begin{tabular}{|l|l|l}
\hline $30 \mathrm{Jcm}^{-2}$ & 3 minutes, 40 seconds & 29 minutes, 30 seconds \\
\hline
\end{tabular}

Table 1: Time to achieve the doses of 9 and $30 \mathrm{Jcm}^{-2}$ for each method of delivery

Figures 2 and 3 provide a visual presentation of the set-up for light energy delivery.

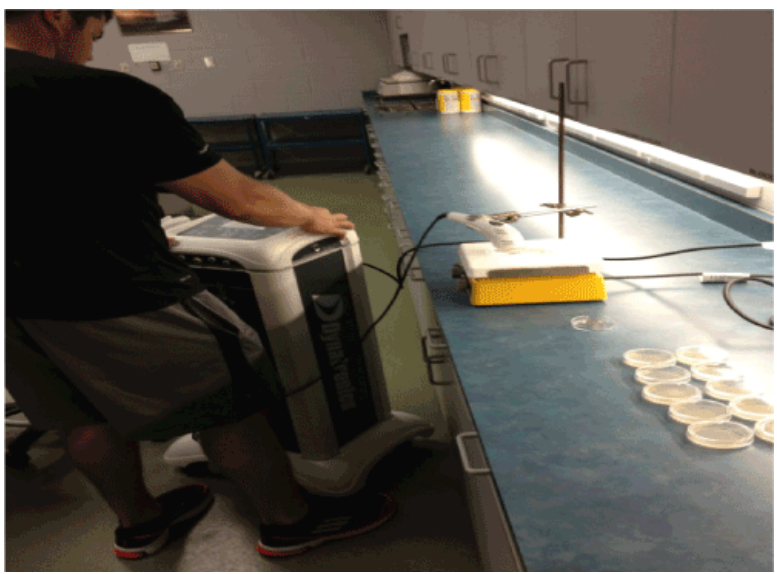

Figure 2: Experimental Set-Up for High Rate of Energy Delivery

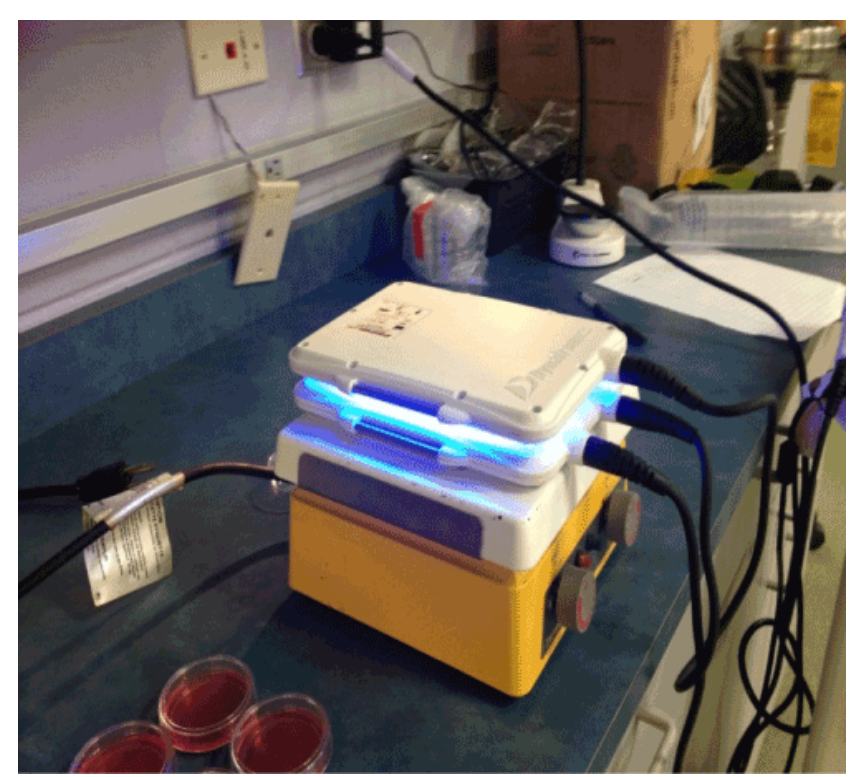

Figure 3: Experimental Set-Up for Low Rate of Energy Delivery

\section{Data Analysis}

Data were analyzed using SPSS 20.0 software. The two independent variables were dose and rate of energy delivery. The dependent variable was kill rate percentage. The data were first subjected to a series of two-tailed, paired student t-tests to determine whether any dose or delivery rate produced a significant kill rate percentage (CFUs in control versus treated). Then a repeated-measures two - way analysis of variance was employed to determine whether any interactive effect (dose and rate) was present. Each of the possible combinations of independent variables was analyzed for its contribution to inhibiting the organism (kill rate percentage) to determine whether an interaction effect was present. A post hoc analysis (Tukey's Honest Significant Difference) was employed where an interaction effect was demonstrated by the two-way ANOVA.

\section{Results}

Table 2 displays the basic outcomes of the study. Three of the four dose/rate combinations produced a significant kill rate for this organism $(\mathrm{p}=0.00)$. Nine $\mathrm{Jcm}^{-2}$ delivered at the high rate $(125$ $\mathrm{mWcm}^{-2}$ ) did not produce a kill rate. In fact, this dose and rate combination, at this combined wavelength of 464 and $850 \mathrm{~nm}$, resulted in actual increased growth compared to untreated controls.

These data demonstrated a significant interactive effect between dose and rate $(\mathrm{F}=194.717, \mathrm{p}=0.00)$. Tukey's HSD revealed that 30 $\mathrm{Jcm}-2$ delivered at the lower rate $\left(20 \mathrm{mWcm}^{-2}\right)$ was significantly more inhibitory than any other dose/rate combination, producing a kill rate of $65.44 \%$.

\section{Discussion}

Pseudomonas aeruginosa is the most frequently isolated bacterium among those gram negative rods that are obligate aerobes (nonfermenters). It is a major cause of nosocomial infections where the source is often contaminated IV fluids, ophthalmic solutions, hydrotherapy tanks, respiratory equipment and even disinfectant solutions such as quaternary ammonium compounds. In the absence of strict hand washing protocols, this organism may be spread from patient to patient by the hands of hospital personnel. Organisms that have colonized the host to become part of the microbiota of the respiratory and gastrointestinal tract are another important source of infection. Five $-10 \%$ of healthy individuals may carry $P$. aeruginosa in their gastrointestinal tract, but that percent increases among hospitalized patients because of the selective pressure of antibiotics.

\begin{tabular}{|c|c|c|c|c|c|c|}
\hline $\begin{array}{l}\text { Dose } \\
\text { (Jcm-2) }\end{array}$ & $\begin{array}{l}\text { Rate } \\
\text { (mWcm-2) }\end{array}$ & $\mathbf{N}$ & Treated $(X \pm S D)$ & $\mathbf{N}$ & Control $(X \pm S D)$ & $p$-value \\
\hline 9 & 125 & 15 & $51.33 \pm 5.61$ & 15 & $47.60 \pm 7.64$ & $0.00+$ \\
\hline 9 & 20 & 15 & 22. $80 \pm 4.69$ & 15 & $47.60 \pm 7.64$ & 0.00 \\
\hline
\end{tabular}




\begin{tabular}{|l|l|l|l|l|l|l|}
\hline 30 & 125 & 15 & $31.13 \pm 5.46$ & 15 & $47.60 \pm 7.64$ & 0.00 \\
\hline 30 & 20 & 15 & $16.46 \pm 2.79$ & 15 & $47.60 \pm 7.64$ & 0.00 \\
\hline
\end{tabular}

+This dose and rate combination did not produce a kill. The treated group saw increased growth versus control.

Table 2: Kill rate by dose and rate of delivery: P. aeruginosa

Infection with $P$. aeruginosa is a major problem in immuno compromised individuals such as patients with skin and soft tissue injuries (i.e. burn victims), intravenous drug users, patients on long term, broad spectrum antibiotics, neutropenic patients, and patients with respiratory diseases, especially cystic fibrosis patients. $P$. aeruginosa infections are particularly dangerous because the organism is frequently resistant to many commonly used antibiotics $[17,18]$.

As mentioned in the introduction, we have found visible and infrared wavelengths to be effective inhibitors of this and other organisms [5-10]. Our work had led us to question whether rate of delivery, and not just dose, might impact the effectiveness of the light energy. Wainwright 16 suggested as much. This current experiment seems to support the importance of delivery rate. We hypothesize that only the light energy absorbed by the organism can have an effect in terms of reactive oxygen formation. It has been postulated that reactive oxygen is critical in terms of inhibition $[19,20]$. When the rate of delivery exceeds the organism's capacity for energy absorption, that excessive energy may have no effect. Our intention for the in vitro studies we have performed is to inform clinical practice. This experiment suggests that if clinicians use light as a decontamination technique to treat wounds, clean instruments, etc., those clinicians should consider rate of energy delivery to improve potential clinical outcomes. More specifically, a lower rate of delivery (approximately 20 $\mathrm{mWcm}^{-2}$ ) may produce a better outcome in the treatment of wounds infected with $P$. aeruginosa.

\section{Conclusions}

A combination of 464 and $850 \mathrm{~nm}$ light energy is an effective inhibitor of $P$. aeruginosa. Dose and rate of delivery are both important factors in terms of enhancing the inhibitory effect in vitro. There is an interactive effect between dose and rate of delivery. At a given dose, lower rates of delivery $\left(20 \mathrm{mWcm}^{-2}\right)$ are more effective than higher rates of delivery.

\section{References}

1. Church D, Elsayed S, Reid O, Winston B, Lindsay R (2006) Burn wound infections. Clin Microbiol Rev 19: 403-434.

2. Dai T, Gupta A, Huang YY, Yin R, Murray CK, et al. (2013) Blue light rescues mice from potentially fatal Pseudomonas aeruginosa burn infection: efficacy, safety, and mechanism of action. Antimicrob Agents Chemother 57: 1238-1245.

3. Doern GV, Heilmann KP, Huynh HK, Rhomberg PR, Coffman SL, Brueggemann AB (2001) Antimicrobial resistance among clinical isolates of Streptococcus pneumonia in the United States during 199-200, including a comparison of resistance rates since 1994-1995. Antimicrob. Agents Chemother. 45:1721-1729.

4. Bush K, Courvalin P, Dantas G, Davies J, Eisenstein B, et al. (2011) Tackling antibiotic resistance. Nat Rev Microbiol 9: 894-896.
5. Guffey JS, Wilborn J (2006) In vitro bactericidal effects of 405-nm and 470-nm blue light. Photomed Laser Surg 24: 684-688.

6. Guffey JS, Wilborn J (2006) Effects of combined 405-nm and 880-nm light on Staphylococcus aureus and Pseudomonas aeruginosa in vitro. Photomed Laser Surg 24: 680-683.

7. Guffey JS, Payne W, James L, Qian Z (2013) Inactivation of Mycobacterium smegmatis following Exposure to 405-Nanometer Light from a Supraluminous Diode Array. WOUNDS 25:131-135.

8. Guffey JS, Payne W, Jones T, Martin K (2013) Rate of Inactivation of Staphylococcus aureus in an In-Vitro, Multiple Stage Application of 405Nanometer Light from a Supraluminous Diode Array. Photomedicine and Laser Surgery 31:179-182.

9. Guffey JS, Payne W, Martin K and Dodson C. Delaying the Onset of Resistance Formation: Effect of Manipulating Dose, Wavelength and Rate of Energy Delivery of 405, 464 and 850-Nanometer Light for Staphylococcus Aureus. Accepted for publication 11/12/13, WOUNDS Journal. Publication date: April 2014.

10. Guffey JS, Payne W, Qian Z, Martin K, James L, Dodson C (2013). Combined 624-nm and $850-\mathrm{nm}$ illumination at low rates leads to enhanced inhibition of Candida albicans. Int J Res Med Sci. 1:333-337.

11. Murdoch LE, Maclean M, Endarko E, MacGregor SJ, Anderson JG (2012) Bactericidal Effects of $405 \mathrm{~nm}$ Light Exposure Demonstrated by Inactivation of Escherichia, Salmonella, Shigella, Listeria, and Mycobacterium Species in Liquid Suspensions and on Exposed Surfaces. Scientific World Journal, doi:10. 1100/2012/137805.

12. Feese E, Ghiladi RA (2009) Highly efficient in vitro photodynamic inactivation of Mycobacterium smegmatis. J Antimicrob Chemother 64: 782-785.

13. Ganz RA, Viveiros J, Ahmad A, Ahmadi A, Khalil A, et al. (2005) Helicobacter pylori in patients can be killed by visible light. Lasers Surg Med 36: 260-265.

14. Dai T, Gupta A, Murray CK, Vrahas MS, Tegos GP, et al. (2012) Blue light for infectious diseases: Propionibacterium acnes, Helicobacter pylori, and beyond? Drug Resist Updat 15: 223-236.

15. Maclean M, MacGregor SJ, Anderson JG, Woolsey G (2009) Inactivation of bacterial pathogens following exposure to light from a 405-nanometer light-emitting diode array. Appl Environ Microbiol 75: 1932-1937.

16. Wainwright M (1998) Photodynamic antimicrobial chemotherapy (PACT). J Antimicrob Chemother 42: 13-28.

17. Henderson JT (2011) Non-fermenting Gram Negative Rods. In: Kiser K, Payne W, Taff T. Clinical Laboratory Microbiology: A Practical Approach Boston, MA Pearson: 537-560.

18. Ashkenazi H, Malik Z, Harth Y, Nitzan Y (2003) Eradication of Propionibacterium acnes by its endogenic porphyrins after illumination with high intensity blue light. FEMS Immunol Med Microbiol 35: 17-24.

19. Hamblin MR, Viveiros J, Yang C, Ahmadi A, Ganz RA, et al. (2005) Helicobacter pylori accumulates photoactive porphyrins and is killed by visible light. Antimicrob Agents Chemother 49: 2822-2827.

20. Maclean M, Macgregor SJ, Anderson JG, Woolsey GA (2008) The role of oxygen in the visible-light inactivation of Staphylococcus aureus. J Photochem Photobiol B 92: 180-184. 